\title{
BCRP/ABCG2 in the Placenta: Expression, Function and Regulation
}

\section{Qingcheng Mao ${ }^{1,2}$}

The following error was published in this article.

In the fourth sentence of the last section ("Clinical Significance of BCRP Expression in the Placenta and Future Direction") —_ "Glyburide is an excellent example of drugs that bypass BCRP-mediated transport by human placenta."___bypass" should be changed to "undergo".

\footnotetext{
${ }^{1}$ Department of Pharmaceutics, School of Pharmacy, University of Washington, P.O. Box 357610 Seattle, Washington 98195-7610, USA.

${ }^{2}$ To whom correspondence should be addressed. (e-mail: qmao@u.washington.edu)
} 